\title{
An experimental study of the effects of lobed nozzles on installed jet noise
}

\author{
Benshuai Lyu' ${ }^{10} \cdot$ Ann P. Dowling²
}

Received: 22 February 2019 / Revised: 8 October 2019 / Accepted: 9 October 2019 / Published online: 11 November 2019

(c) The Author(s) 2019

\begin{abstract}
Jet noise remains a significant aircraft noise contributor, and for modern high-bypass-ratio aero-engines the strong interaction between the jet and aircraft wing leads to intensified installed jet noise. An experiment is carried out in this paper to study the effects of lobed nozzles on installed jet noise. It is found that the lobed nozzles, compared to round nozzles, have similar effects on installed jet noise for all the plate positions and Mach numbers tested. On the shielded side of the plate, the use of lobed nozzles leads to a noise reduction in the intermediate- and high-frequency regimes, which is thought to be due to a combination of enhanced jet mixing and more effective shielding effects by the flat plate. On the reflected side of the plate, noise reduction is only achieved in the intermediate frequency range; the little noise reduction or a slight noise increase observed in the high-frequency regime is likely due to enhanced jet mixing. On both sides of the plates, little noise reduction is achieved for the low-frequency noise due to the scattering of jet instability waves. This is likely to be caused by the fact that lobed nozzles cause negligible change to the dominant mode 0 (axisymmetric) jet instability waves. That the jet mean flow quickly becomes axisymmetric downstream of the jet exit could also play a role.
\end{abstract}

\section{Graphic abstract}

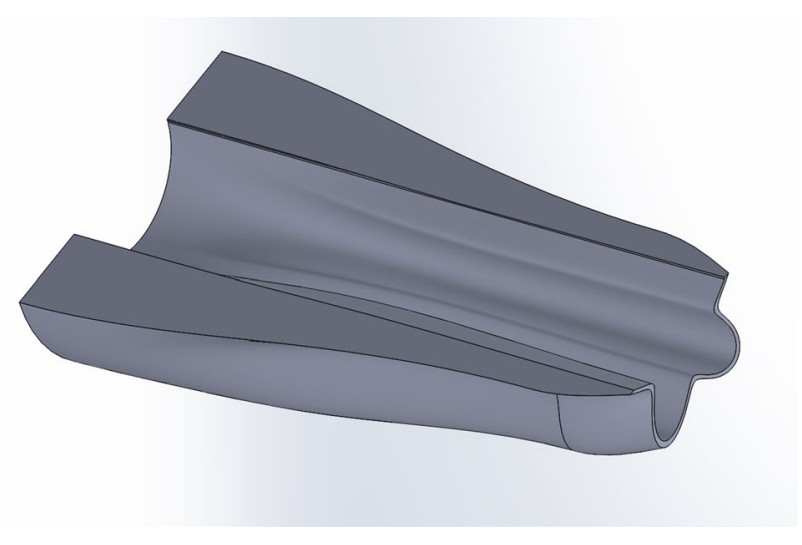

Benshuai Lyu

bl362@cam.ac.uk

1 Department of Engineering (now at Department of Applied Mathematics and Theoretical Physics), University of Cambridge, Cambridge, UK

2 Department of Engineering, University of Cambridge, Cambridge, UK

\section{Introduction}

Aviation has seen a rapid expansion for the past few decades, and it is expected to continue to do so for the next 2 decades. The environmental impacts of air travel are becoming increasingly important (Mahashabde et al. 2011). Among them, aircraft noise is now a matter of considerable public concern because of its wide range of health-related consequences (Huss et al. 2010; Münzel et al. 2016; Beutel et al. 2016). Aircraft noise consists of many sources, such 
as airframe noise, engine fan noise, combustion noise, jet noise, etc.

Jet noise remains a significant noise source when an aircraft takes off, but its reduction poses a great challenge. Part of the reason lies in the difficulty of accurately modelling turbulence, which is known to be the source of jet noise. However, it has been known for a long time that the power of subsonic jet noise scales as the eighth power of the jet Mach number (Lighthill 1952, 1954). Consequently, for the past few decades the primary and perhaps the most effective noise reduction method has been to reduce the jet exit velocity by increasing aero-engine diameters. This strategy has successfully led to a reduction of aircraft noise by around 20 $\mathrm{dB}$ (Casalino et al. 2008). However, the increasingly large engine diameter leads to a narrow gap between the aeroengine and the aircraft wing (Lyu and Dowling 2017). Jet noise is significantly amplified at low-frequencies because of this close installation, a phenomenon known as the installation effect (Bushell 1975; Head and Fisher 1976; Way and Turner 1980; Shearin 1983). Jet noise measured under such a condition is often referred to as installed jet noise, in contrast to the isolated jet noise (Lyu et al. 2017) studied extensively in the literature (Lighthill 1954, 1963; Tam 1998; Tam and Viswanathan 2008; Jordan and Colonius 2013; Karabasov 2010).

Recent studies have shown that the low-frequency noise intensification observed in installed jets is due to the scattering of jet instability waves (Lyu and Dowling 2016, 2017, 2018a; Cavalieri et al. 2014). The understanding of this mechanism is important in developing new noise reduction methods. For example, it was recently proposed by Piantanida et al. (2016) that installed jet noise could be reduced when an aircraft wing with a swept trailing edge is used. Their experimental results showed that while noise reduction could be observed on one side of the jet (for example on the side of the wing tip), virtually no sound reduction could be observed on the other side (the side close to the wing root). This dependence of sound reduction on the observer locations was explained in the recent work of Lyu and Dowling (2019). They showed that the noise reduction achieved on one side is due to the destructive interference of the scattered pressure along the trailing edge of the swept wing. However, this interference is only significant on one side of the jet, hence the results observed in Piantanida et al.'s experiment.

The identified mechanism of installed jet noise helps to develop alternative noise reduction methods. In particular, since noise is generated because of the scattering of jet instability waves by the trailing edge of the aircraft wing, a straightforward alternative approach is to suppress the instability waves in the first place. Use of chevron nozzles represents one such approach. Chevrons have been known to be able to enhance jet mixing and reduce isolated jet noise (Bridges and Brown 2004; Zaman et al. 2011) at low frequencies but may also lead to noise increase at high frequencies. However, it is worth mentioning that chevron nozzles also change the directivity of the jet noise, hence making it possible to reduce the overall aircraft noise by carefully exploiting the effective shielding effects of aircraft wings using engine-on-top configurations. The current understanding of the low-frequency noise reduction achieved using chevrons is that these chevrons can reduce the large coherence structures originating from the jet instability waves. It is, therefore, of interest to the research community to understand how installed jet noise is affected by chevrons. A recent study was carried out by Bastos et al. (2017) to examine the effects of chevron nozzles on installed jet noise. The results were compared with the installed jet noise spectra for a round nozzle. It was found that when the flat plate, used as a simplified aircraft wing, was placed sufficiently far away from the jet, the chevron nozzle could reduce installed jet noise at all frequencies on the shielded side of the flat plate. However, when the plate was closely integrated with the jet, the low-frequency reduction provided by chevron nozzles became negligible. Moreover, an increasingly pronounced noise increase at high frequencies was observed.

Another alternative method seeking to control installed jet noise by modifying jet instability waves is to use lobed nozzles. The recent work by Lyu and Dowling (2018b) shows that the lobed structure of a jet can indeed change the characteristics of instability waves, including both the temporal growth rate and convection velocity. These changes, however, depend on the azimuthal orders of the instability waves, the number of lobes and their penetration ratio. But the effects of lobed nozzles on installed jet noise are not known. It is worth noting that Tam and Zaman (2000) and Zaman et al. (2003) have examined the isolated jet noise from tabbed and extremely strong lobed nozzles; however, the literature on this is sparse and the effect of nozzle geometry on isolated jet noise is not very well understood, let alone how it affects installed jet noise. Therefore, in this paper, we perform an experimental study to advance our understanding of this, by studying the effects of lobed nozzles on both isolated and installed jet noise.

This paper is structured as follows: Sect. 2 describes the experimental setup, while Sect. 3 shows the results of this experimental study. Section 4 discusses the experimental results, taking the effects of lobed nozzles on jet instability waves into account. The last section summaries the paper and lists future work.

\section{Experimental setup}

The schematic illustration of the experimental setup is shown in Fig. 1. The experiment is carried out in the anechoic chamber at the Cambridge University Engineering 


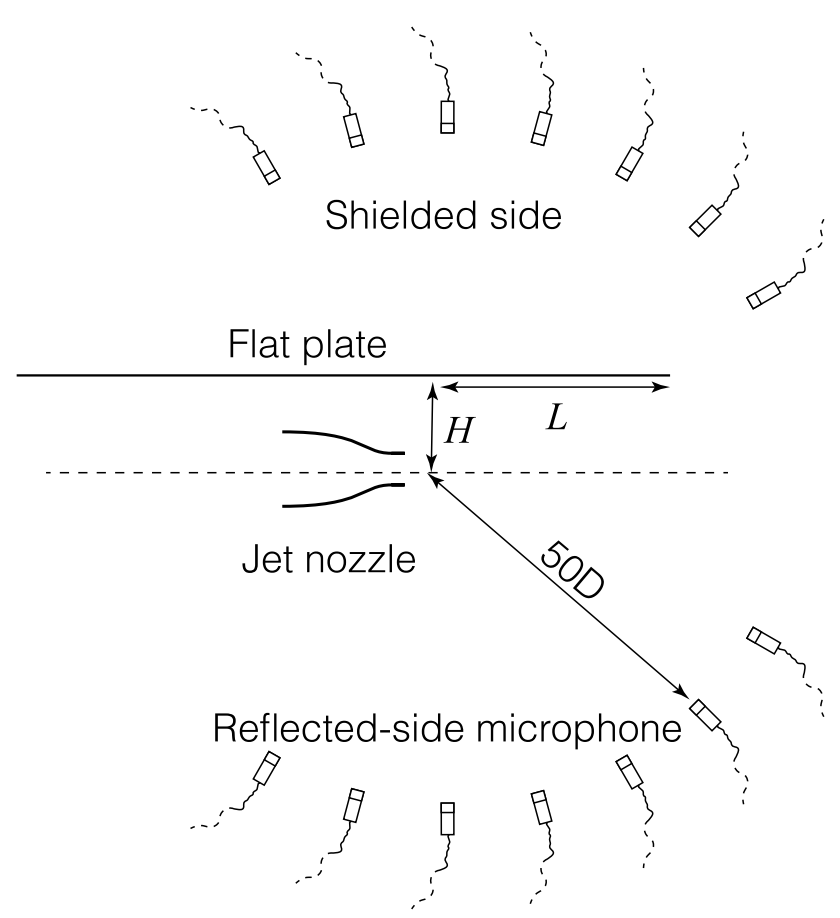

Fig. 1 Schematic illustration of the experimental setup. The experiment carried out in an anechoic chamber with a cut-off frequency of around $200 \mathrm{~Hz}$. The flat plate has a dimension of $12 D \times 24 D$, where $D$ is the jet diameter

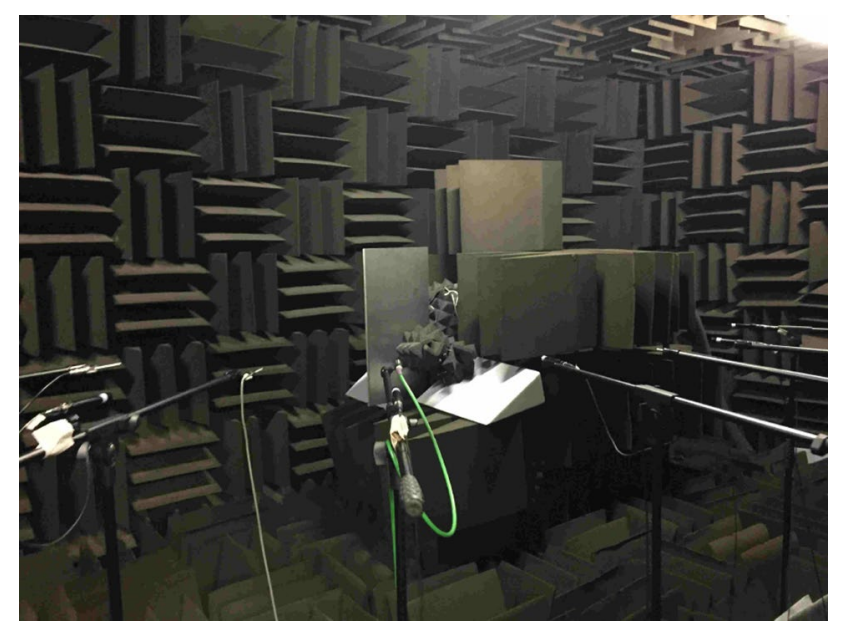

Fig. 2 The installed jet noise experiment setup: microphones are located at $50 D$ to the jet nozzle center on the shielded side, with observer angle ranging from $30^{\circ}$ to $120^{\circ}$ to the jet centerline. The nozzle is behind the rectangular flat plate. The plate is then uninstalled and mounted on the other side of the jet nozzle to measure the noise spectra on the reflected side

Department. The chamber has a lowest operating frequency of around $200 \mathrm{~Hz}$. A photograph of the experimental rig inside the anechoic chamber can be found in Fig. 2.
Table 1 Test parameters for studying the effects of varying $H$ and $L$ on installed jet noise. For each test configuration both RNO0 and LN53 nozzles are tested, and the jet is operated at both $M_{0}=0.5$ and 0.7. In total, 24 installed jet noise tests are conducted

\begin{tabular}{lllll}
\hline Test no. & Nozzles & Mach numbers & $H$ & $L$ \\
\hline 1 & RN00/LN53 & $0.5 / 0.7$ & $3 D$ & $6 D$ \\
2 & RN00/LN53 & $0.5 / 0.7$ & $2 D$ & $6 D$ \\
3 & RN00/LN53 & $0.5 / 0.7$ & $1.5 D$ & $6 D$ \\
4 & RN00/LN53 & $0.5 / 0.7$ & $2 D$ & $4 D$ \\
5 & RN00/LN53 & $0.5 / 0.7$ & $1.5 D$ & $4 D$ \\
6 & RN00/LN53 & $0.5 / 0.7$ & $1.25 D$ & $4 D$ \\
\hline
\end{tabular}

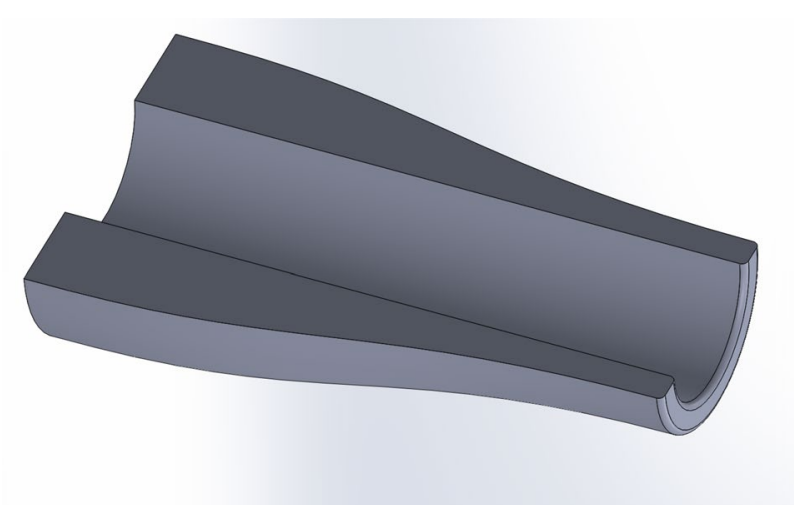

Fig. 3 The reference round nozzle RN00 used in the experiment with a diameter of $2.54 \mathrm{~cm}$

As shown in Fig. 1, 7 GRAS 46BE microphones are placed at $50 D$, where $D$ is the exit diameter of the reference round nozzle, to the center of the jet nozzle at angles in the range of $30^{\circ}$ and $120^{\circ}$ to the jet centerline on either the reflected or the shielded sides. These microphones have a sufficiently flat frequency response up to $80 \mathrm{kHz}$. The electrical signals from these microphones are conditioned, amplified, and then digitalized at a sampling frequency of $120 \mathrm{kHz}$ simultaneously using the VIPER data acquisition system (IMC Ltd). As can be seen in Fig. 1, a flat plate of $12 D \times 24 D$, as a simplified aircraft wing, is placed nearby the jet. The trailing edge of the plate is at $L$ downstream of the jet nozzle, and the separation distance between the jet and the plate is $H$. To obtain a comprehensive database on jet installation effects, both $H$ and $L$ will be varied systematically (Table 1).

As the aim of this study is to investigate the effects of lobes on installed jet noise, a reference round nozzle RN00, shown in Fig. 3, is first used as reference. The nozzle is 3D printed with a resolution of $0.1 \mathrm{~mm}$. The lip of the round nozzle, as can be seen from Fig. 3, has an uncharacteristically large wall thickness because of structural sturdiness considerations. The round nozzle used in this experiment 
has a diameter $D=2.54 \mathrm{~cm}$. The lobed nozzle used in this experiment has an exit profile of

$\sigma=a_{\epsilon}(1+\epsilon \cos N \phi)$,

where $\phi$ is the azimuthal angle, $N$ is the number of lobes, $\epsilon$ is the lobe penetration ratio and $\sigma$ is the radius of the nozzle at azimuthal angle $\phi$. The constant $a_{\epsilon}$ is chosen for specific $\epsilon$ and $N$ (in fact it is independent of $N$ when $N \neq 0$ ) such that the lobed nozzle has the same exit area as the reference round nozzle. The lobed nozzle LN53 used in this experiment has five lobes and a penetration ratio of 0.3 , as shown in Fig. 4. From the section view of the nozzle shown in Fig. 4, one can see that the lobed structure is not formed abruptly at the nozzle exit, but rather through a continuously smooth morphing. The morphing length is around $4 D$ long. The lobed nozzle exit is tapered to have a thickness of $2 \mathrm{~mm}$, as can be seen from Fig. 4 .

To compare the installed jet noise spectra from the lobed nozzle with those from the round nozzle under similar jet operating conditions, the average acoustic Mach number $M_{0}$ is kept to be the same. Note $M_{0}$ is defined via $M_{0}=U_{j} / c_{0}$, where $U_{j}$ is averaged jet velocity calculated from jet mass flow rate and $c_{0}$ is the ambient speed of sound. The velocity profile for the lobed nozzle is expected to be slightly more non-uniform than that for the round nozzle, so the thrust may change. Early studies show that although drastically lobbed nozzles with inclined angles may result in a small thrust loss (Tam and Zaman 2000; Zaman et al. 2003), moderately lobbed nozzles produce neither significant improvement nor significant degradation in thrust efficiency (Lopera et al. 2006). Given that the nozzle LN53 is moderately lobed without an inclined angle, we expect the thrust change to be negligible, and a direct comparison of the noise spectra should, therefore, be a fair comparison. In order to have a comprehensive database, the values of $H$ and $L$ are subsequently varied systematically. As can be seen from Table 1, for each

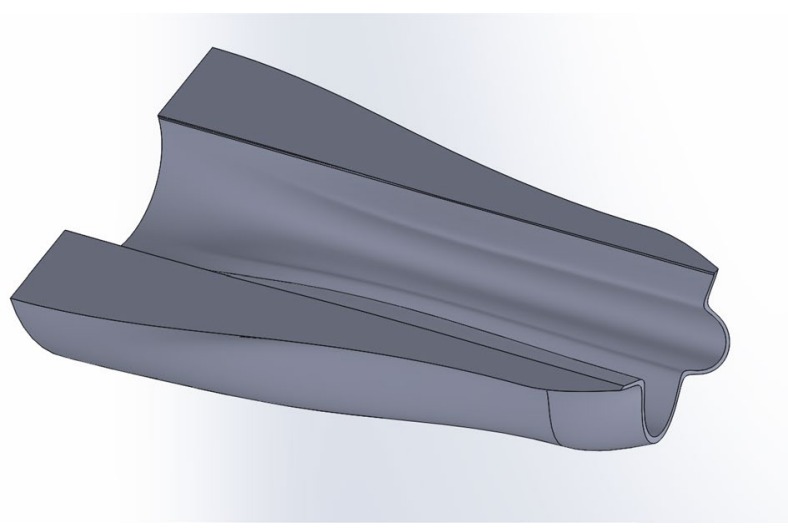

Fig. 4 The lobed nozzle LN53 used in the experiment: it has five lobes with a penetration ratio $\epsilon=0.3$ test configuration, results are obtained for both the round and lobed nozzles, and the jet is operated first at $M_{0}=0.5$ and then $M_{0}=0.7$. Together with isolated jet noise measurement for both nozzles, 28 tests are conducted in total.

\section{Results}

Before discussing experimental results, we validate the current experimental data against others' published in the open literature. We choose to compare the reference isolated jet noise spectra with those obtained by Tanna (1977). This has been performed in an earlier work of the authors (Lyu and Dowling 2018a). Suffice to mention here that although the Reynolds number in Tanna's study is twice as large as that used in our study, the agreement between the two is good enough to show that the measurement is reliable. Details of this validation can be found in Lyu and Dowling (2018a). In what follows, we will start by showing the results for isolated jet noise. The effects of lobes on installed jet noise is presented subsequently for $M_{0}=0.5$ and 0.7 , respectively.

\subsection{Effects of lobes on isolated jet noise}

The effects of lobed nozzles on isolated jet noise spectra can be seen from Fig. 5. Figure 5a shows the noise spectra of both the round and lobed nozzles at Mach number 0.5. Before discussing these results, it is worth emphasizing again that the flow rates are kept the same for both the round and lobed nozzles. Compared with the isolated spectra of the round nozzle, a noise reduction of around 1.5-2 $\mathrm{dB}$ is achieved for lobed jets except for an observer at $30^{\circ}$ to the jet axis. The noise reduction is most evident in the intermediate-frequency regime, i.e. $500 \mathrm{~Hz}<f<5000 \mathrm{~Hz}$, where $f$ is the frequency. The noise reduction is in the lowfrequency regime $(f<500 \mathrm{~Hz})$ is negligible. In the highfrequency regime $(f>8000 \mathrm{~Hz})$ there is a slight noise increase, which is more evident at $90^{\circ}$. This is similar to what was observed for chevron jets (Bridges and Brown 2004). It is known that chevron nozzles enhance jet mixing and lead to a faster decay of centerline velocities, which results in a reduction of low-frequency and an increase of high-frequency jet noise (Bridges and Brown 2004; Zaman et al. 2011). The high-frequency noise increase observed for the lobed nozzle in this experiment is likely to be due to the same effects.

The noise reduction using lobed nozzles is more evident for higher-speed jets. This can be seen from Fig. 5b, where the jet Mach number is 0.7. An average noise reduction of $3 \mathrm{~dB}$ is observed at all observer angles, including $30^{\circ}$. The noise reduction again is only pronounced in the intermediate-frequency regime $(500 \mathrm{~Hz}<f<10,000 \mathrm{~Hz})$ with little noise increase at high frequencies $(f>10,000 \mathrm{~Hz})$. 
(a)

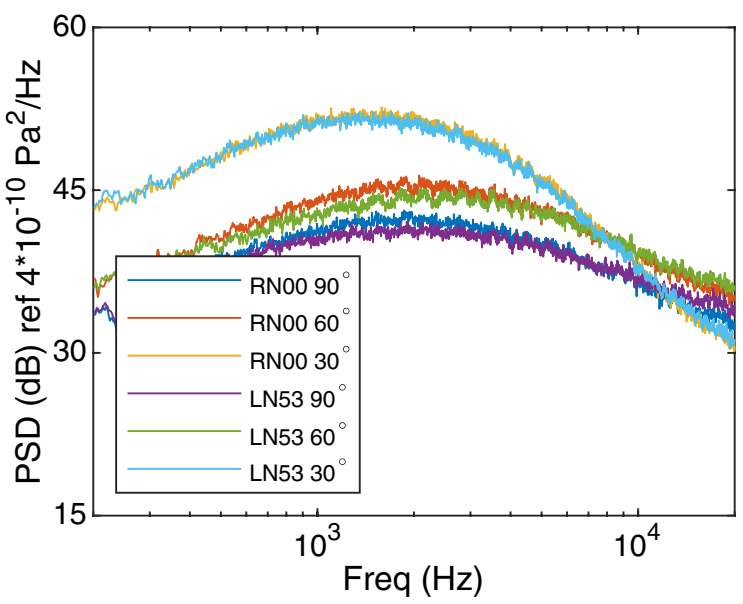

(b)

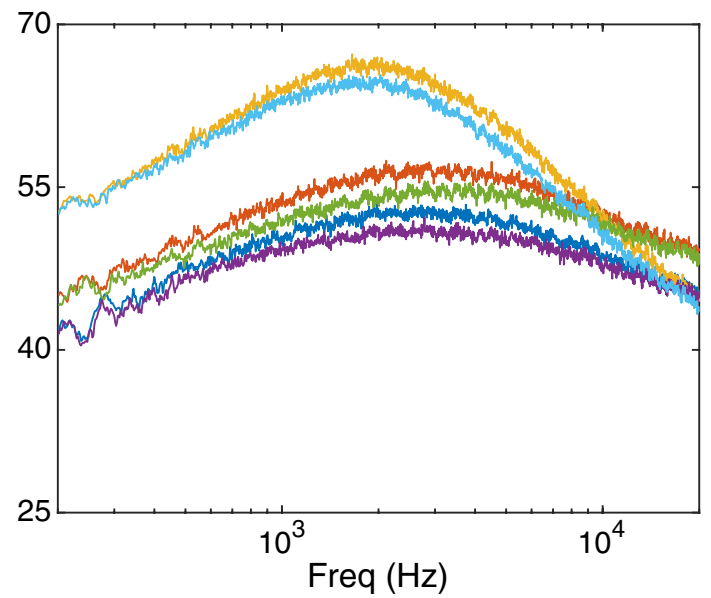

Fig. 5 Comparison of the isolated jet noise between round and lobed nozzles: $\mathbf{a} M_{0}=0.5 ; \mathbf{b} M_{0}=0.7$

These results show that the lobed nozzle has similar effects as chevron nozzles, both of which result in a noticeable isolated jet noise reduction at intermediate frequencies and a slight or negligible noise increase at high frequencies. The high-frequency penalty is reduced for higher Mach numbers.

\subsection{Effects of lobes on installed jet noise at $M_{0}=0.5$}

To show the effects of nozzle lobes on installed jet noise, we compare the installed jet noise spectra for the round and lobed nozzles. The spectra at $M_{0}=0.5$ are presented first in Figs. 6 (on the shielded side) and 7 (on the reflected side). Before discussing the results, we note that the low-frequency humps observed in these spectra are due to the scattering of instability waves, while the high-frequency noise is due to jet mixing. The actual frequency ranges depend on the positions of the plate, for example the low-frequency humps refer to $200 \mathrm{~Hz}<f<1000 \mathrm{~Hz}$ in Fig. 6a and $200 \mathrm{~Hz}<f<2000 \mathrm{~Hz}$ in Fig. 6b.

Figure 6a shows the comparison when the plate's trailing edge is at $L=6 D$ and $H=3 D$. One can see that an average $1.5 \mathrm{~dB}$ noise reduction is achieved for all observer angles in the intermediate- and high-frequency regimes $(f>1000 \mathrm{~Hz})$. This is likely due to the enhanced jet mixing which also results in a reduction of isolated jet noise (see Fig. 5a). But note that no sound increase is found at high frequencies, whereas an increase is observed for isolated jet noise, as shown in Fig. 5a. This is similar to the results for chevron nozzles (Bastos et al. 2017). The fact that no sound increase is observed due to the use of chevron and lobed nozzles at high frequencies for installed jet noise, as opposed to isolated jet noise, could be caused by the enhanced noise shielding effects by the flat plate when lobed and chevron jets are used. It is known that chevrons enhance jet mixing and, therefore, result in a shorter jet mean-flow potential core. Similar effects are expected for lobed nozzles. This would make the shielding effects of the flat plate more effective. Therefore, although chevron and lobed nozzles may generate more noise at high frequencies, this is outweighed by the more effective shielding effects. This explains why there is a consistent noise reduction in the intermediate- and high-frequency regimes. However, little sound reduction is observed at low frequencies $(f<1000 \mathrm{~Hz})$, especially around the low-frequency hump. This shows that although lobed nozzles can result in a noise reduction in the intermediate- and high-frequency regimes, they cause little change to the low-frequency installed jet noise. Since the low-frequency noise enhancement results from the scattering of jet instability waves, this suggests that the lobed nozzle has little effect on this scattering contribution to jet noise.

Moving the plate closer to the jet axis to $H=2 D$, while $L$ is kept at $6 D$, results in, as expected, stronger noise enhancement at low frequencies, as shown in Fig. 6b. The trend that a slight noise reduction is observed only at intermediate and high frequencies remains. The same observations can be made for all other plate positions, see Fig. $6 \mathrm{c}-\mathrm{f}$.

The comparison of noise spectra on the other side of the plate is shown in Fig. 7. Figure 7a shows the results for $L=6 D$ and $H=3 D$, from which we can see that the effects of lobed nozzles are slightly different from those on the other side of the plate. In particular, although noise reduction is achieved in the intermediate frequency regime $(1000 \mathrm{~Hz}<f<5000 \mathrm{~Hz})$, a slight noise increase is also observed in the high-frequency range $(f>8000 \mathrm{~Hz})$. This is different from the situation on the shielded side, where noise reduction persists within the entire mid- to high-frequency regime $(f>1000 \mathrm{~Hz})$. The noise increase observed on the reflected side at high frequencies is likely 
(a)

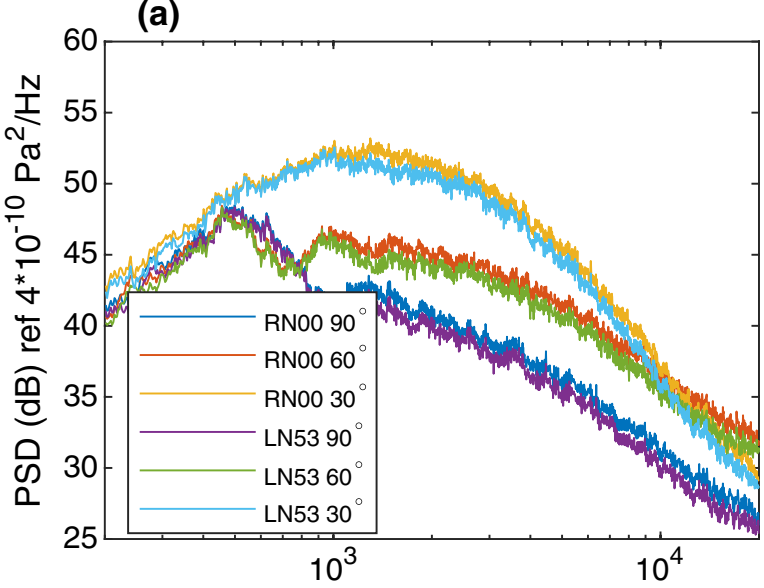

(c)

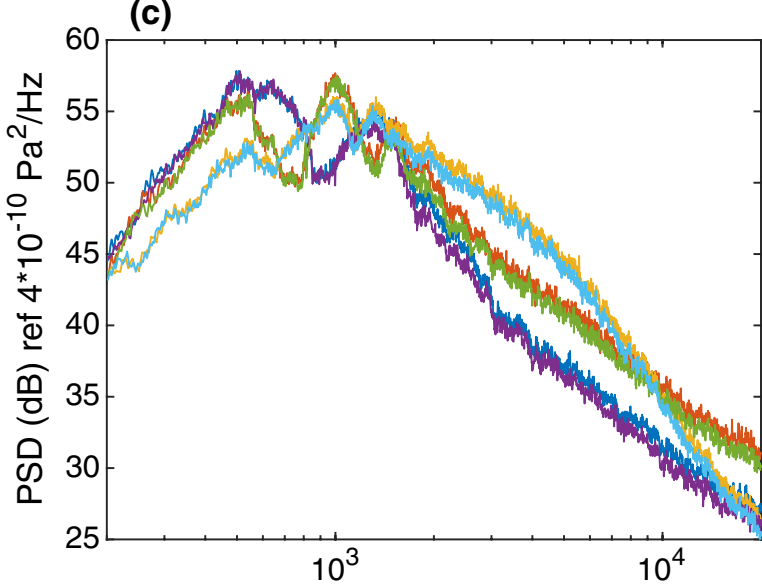

(e)



(b)

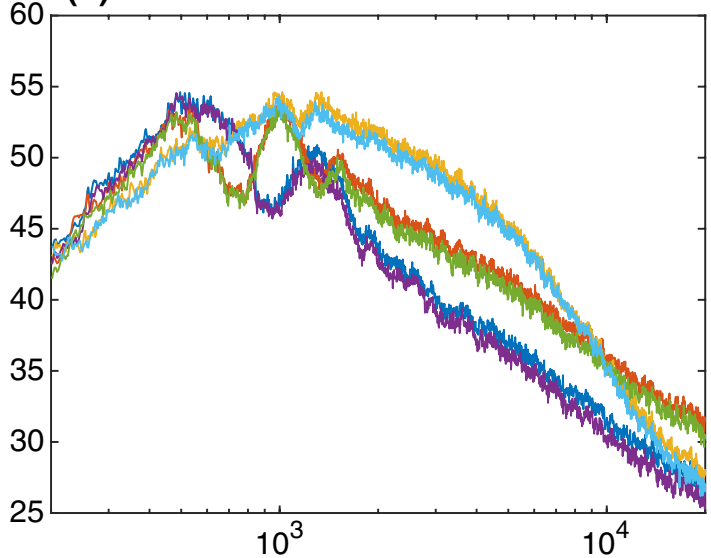

(d)

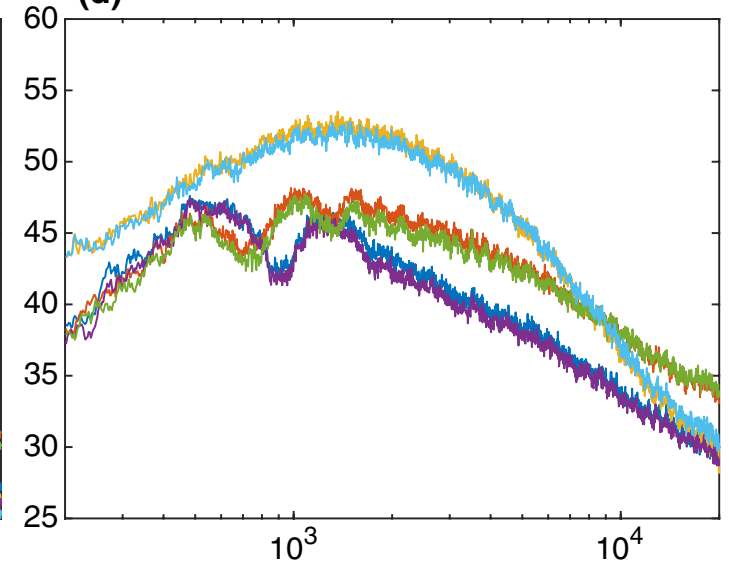

(f)

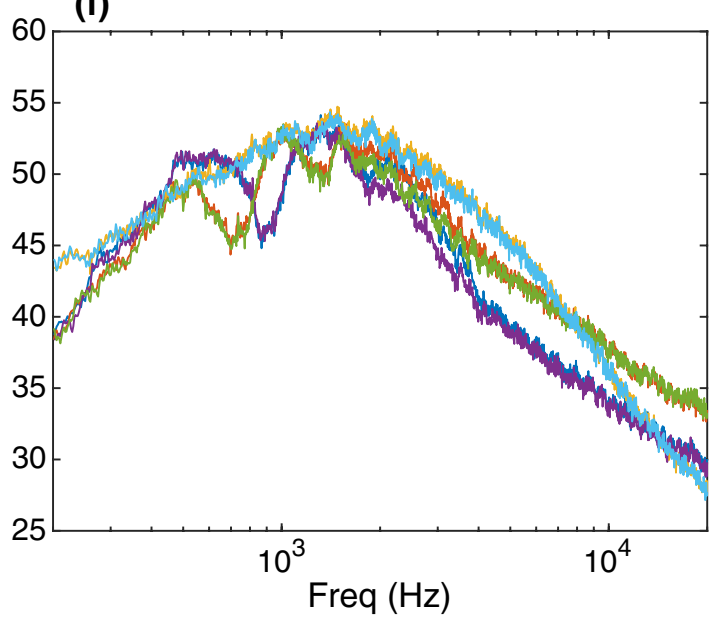

Fig. 6 Installed noise spectra of the round and lobed jets on the shielded side for various plate positions at the Mach number of 0.5 : a $L=6 D, H=3 D ; \mathbf{b} L=6 D, H=2 D ; \mathbf{c} L=6 D, H=1.5 D ; \mathbf{d} L=4 D, H=2 D ; \mathbf{e} L=4 D, H=1.5 D ; \mathbf{f} L=4 D, H=1.25 D$

due to the enhancement of jet mixing. This is likely to be true especially considering that this noise increase occurs in the same frequency range as that of the isolated jet ( $f>8000 \mathrm{~Hz}$ ), as shown in Fig. 5a. On the reflected side, the plate does not shield the noise and the increased highfrequency jet noise due to enhanced mixing propagates to the far field. On the shielded side, this noise increase is outweighed by enhanced shielding and a noise reduction is achieved instead. Hence different behaviour is obtained on the two observer sides. Changing the plate positions does not change these tendencies, as can be seen from Fig. 7b-f. 
(a)

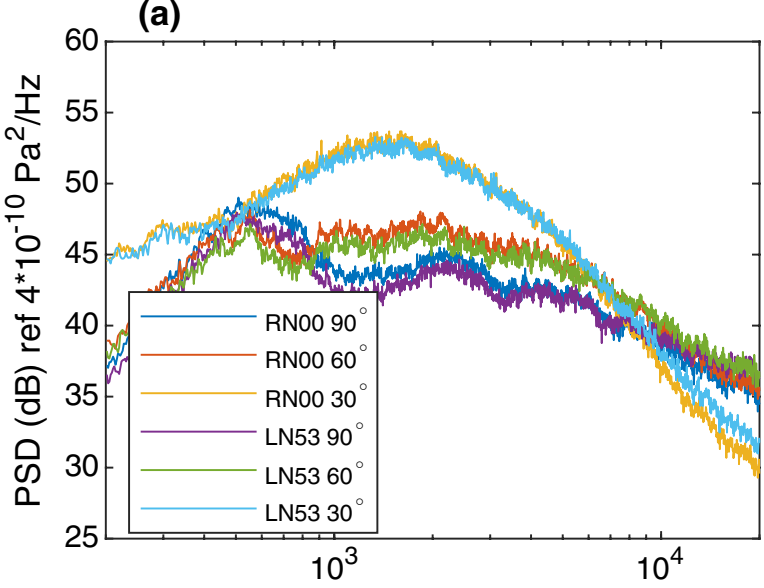

(c)

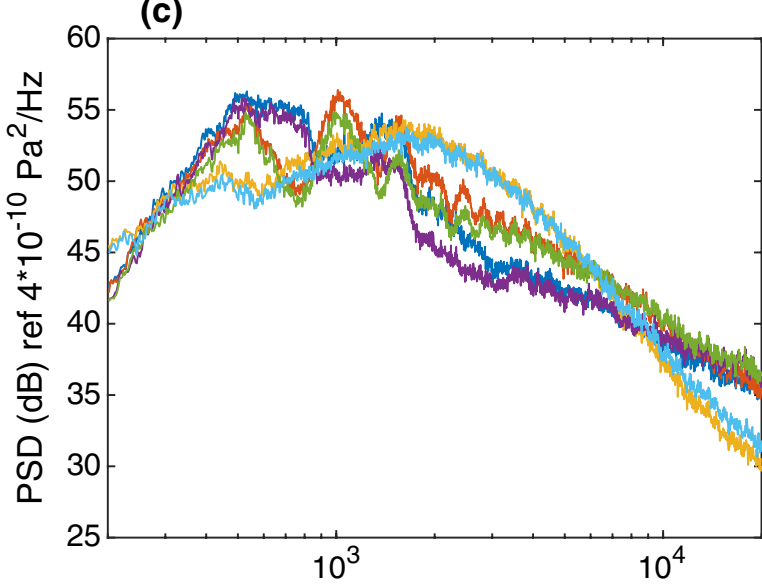

(e)

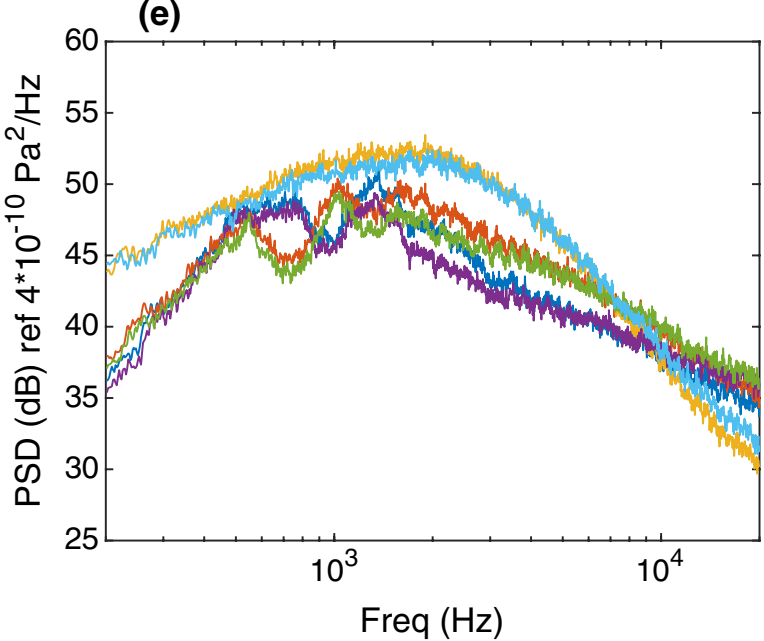

(b)

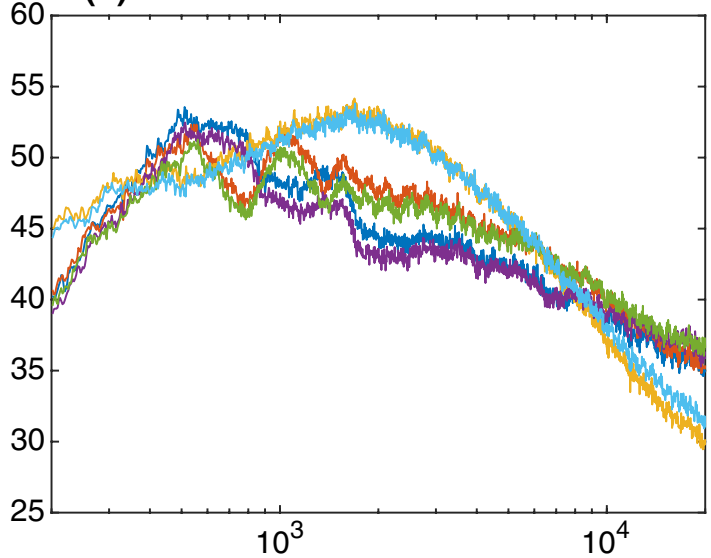

(d)

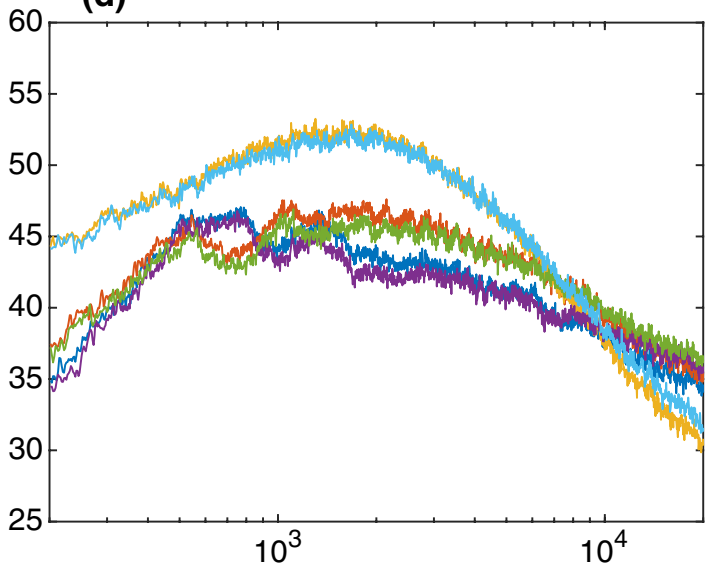

(f)

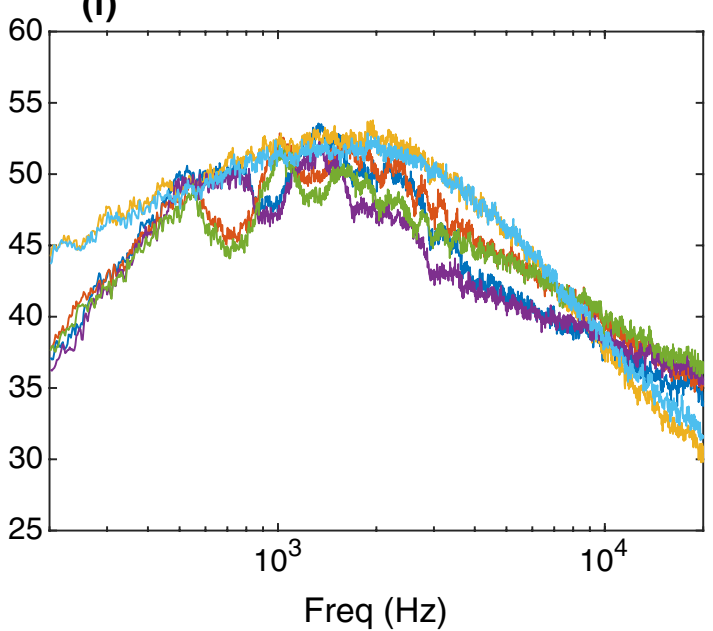

Fig. 7 Installed noise spectra of the round and lobed jets on the reflected side for various plate positions at the Mach number of 0.5: a $L=6 D, H=3 D ; \mathbf{b} L=6 D, H=2 D ; \mathbf{c} L=6 D, H=1.5 D ; \mathbf{d} L=4 D, H=2 D ; \mathbf{e} L=4 D, H=1.5 D ; \mathbf{f} L=4 D, H=1.25 D$

At low frequencies $(f<1000 \mathrm{~Hz})$, the low-frequency humps do not change significantly, although compared to those on the shielded side, there is a marginal noise reduction of around $1 \mathrm{~dB}$ in Fig. $7 \mathrm{a}-\mathrm{c}$. It is possible that this is caused by the different jet refraction profiles between the round and lobed jets - the noise due to instability wave scattering is refracted by the jet mean flow before reaching the observer on the reflected side. The jet mean flows have different velocity profiles between the round and lobed nozzles, hence different effects on the noise amplitude. This jet refraction, however, does not occur on the shield side, and this difference may account for the different trends 
observed between the shielded and reflected sides. If this is the cause, we would expect a less pronounced difference when $L$ is smaller, because the jet plume has a smaller size in the upstream location. This is indeed the case as shown in Fig. 7d-f.

\subsection{Effects of lobes on installed jet noise at $M_{0}=0.7$}

Figure 6 shows that the use of this lobed nozzle does not change the installed jet noise spectra at low frequencies. However, it does result in a slight noise reduction at intermediate and high frequencies $(f>1000 \mathrm{~Hz})$. On the other side of the plate, however, the noise reduction is more effective in the intermediate frequency regime $(1000 \mathrm{~Hz}<f<5000 \mathrm{~Hz})$, while a slight noise increase is observed at high frequencies $(f>8000 \mathrm{~Hz})$. There is also a slight noise reduction at low frequencies, possibly caused by the refraction effect of jet plumes. Does this trend still hold for higher Mach numbers? The answer can be found in Figs. 8 and 9, where the comparisons of the installed noise spectra between the round and lobed nozzles at a Mach number of 0.7 are shown.

Figure 8 shows the comparison of the noise spectra on the shielded side. One can clearly see that a noise reduction of up to $4 \mathrm{~dB}$ is achieved at intermediate and high frequencies $(f>1000 \mathrm{~Hz})$, while little noise reduction occurs at low frequencies $(f<1000 \mathrm{~Hz})$. This tendency generally remains to be true for all different plate positions (see Fig. 8a-f). In the preceding section, we have speculated that the noise reduction observed at high frequencies is caused by an enhanced jet mixing and a more effective noise shielding provided by the flat plate. This presumption is now further supported by the spectra observed for the $M_{0}=0.7$ jets. This is consistent with the amount of noise benefit observed from isolated jets due to intensified mixing, e.g. the noise reduction of the isolated spectra due to the enhanced mixing is around $3 \mathrm{~dB}$ at a Mach number of 0.7 , which is similar to the $4 \mathrm{~dB}$ observed for installed jets.

The comparison of installed jet noise spectra for $M_{0}=0.7$ on the reflected side of the plate is shown in Fig. 9. A maximum of $4 \mathrm{~dB}$ noise reduction is observed, but only in the intermediate-frequency regime $(1000 \mathrm{~Hz}<f<10,000 \mathrm{~Hz})$, and this noise reduction diminishes at high frequencies. This trend is similar to that observed in Fig. 7. In contrast to Fig. 8, we also see a slight noise reduction at low frequencies nearby the hump $(f \sim 800 \mathrm{~Hz})$. As we mentioned in Sect. 3.2, this is likely to be caused by the refraction of jet plumes.

In summary, the effects of the lobed nozzle on installed jet noise are nearly identical for all plate positions and Mach numbers: on the shielded side of the flat plate, lobed nozzles do not noticeably change the installed jet noise spectra at low frequencies. However, it does result in a slight noise reduction at intermediate and high frequencies. This is thought to be caused by the combination of enhanced jet mixing and more effective shielding by the flat plate. On the reflected side of the flat plate, the flat plate does not shield noise any more, and a slight or negligible noise increase is therefore observed at the very high frequencies. In addition, the noise due to the scattering of instability waves has to pass through the jet plume in order to reach the observer. Since the jet plumes of the round and lobed nozzles are different, this causes a very slight change to the effects of lobed nozzles compared to those on the shielded side, especially at low frequencies.

\section{Discussion of the experimental results}

As mentioned in Sect. 1, the earlier work of the authors (Lyu and Dowling 2018b) shows that the stability characteristics of base flows of a lobed vortex-sheet type jet are different from those of an axisymmetric jet. The differences consist of changes to both the convection velocity and the temporal growth rate of instability waves. These changes become more pronounced as the number of lobes $N$ and the penetration ratio $\epsilon$ increase. However, instability waves of different orders are affected differently by the lobe geometry. In particular, little change occurs for mode 0 (axisymmetric mode), no matter how large both $N$ and $\epsilon$ are. On the other hand, an evident alteration of the characteristics of highorder jet instability waves occurs when $N>1$. For $N=3$ and $N=5$, azimuthally even and odd instability waves demonstrate the same characteristics. However, for $N=2$ and $N=1$, even and odd instability waves of lobed jets exhibit two different types of behaviour, with one having favourable effects on reducing installed jet noise and the other having adverse. Therefore, for the sake of suppressing instability waves, or achieving installed jet noise reduction, it is desired to use a lobed profile of large $N$, such as $N=5$, with a large penetration ratio.

In Sect. 3, we see that the use of lobed nozzles does not notably change installed jet noise at low frequencies, which is due to the scattering of jet instability waves. Combined with the stability results concluded in the earlier work, as described above, we may discuss the possible causes.

Since only the nozzle LN53 is tested in this experiment, we focus on the stability characteristics of a lobed jet with $N=5$ and $\epsilon=0.3$. According to the earlier work, the mode 0 jet instability waves of a lobed jet of a vortexsheet type are not sensitive to the lobed geometry of $N=5$ and $\epsilon=0.3$. The changes in both the convection velocity and temporal growth rate are negligible. This is especially true for low frequencies, where installed jet noise is relevant (see Figure 7 in Lyu and Dowling 2018b for example). Therefore, although higher-order instability waves could 
(a)

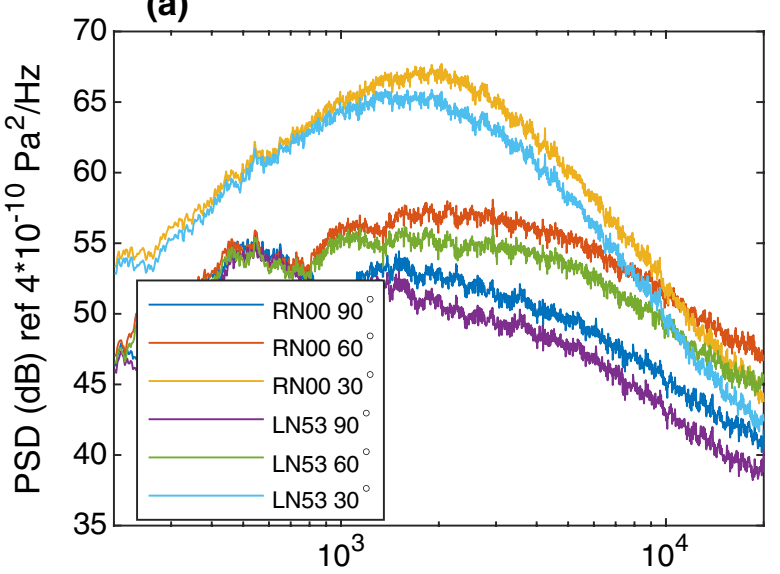

(c)

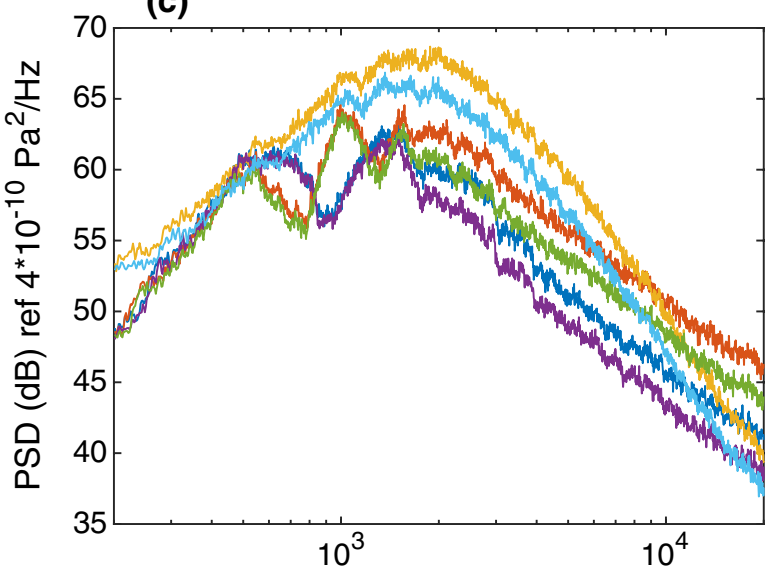

(e)



(b)

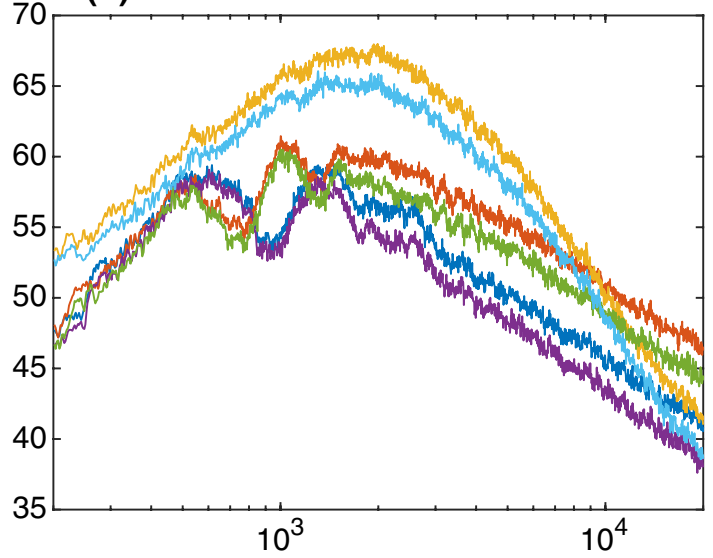

(d)

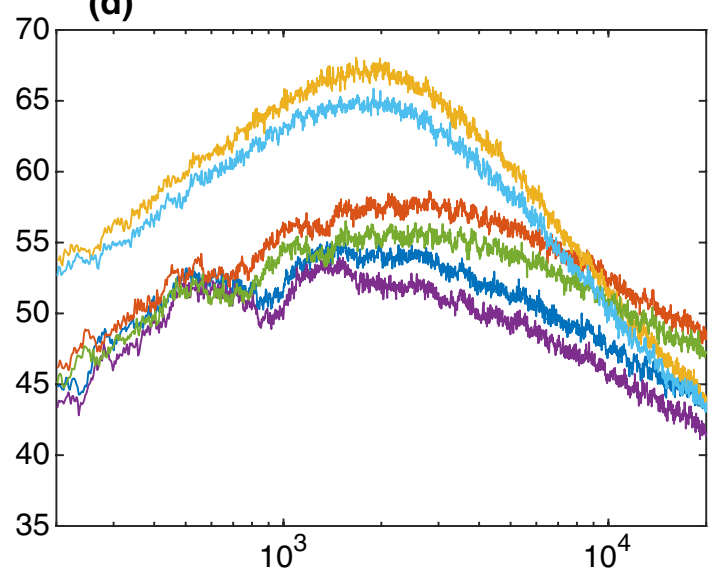

(f)

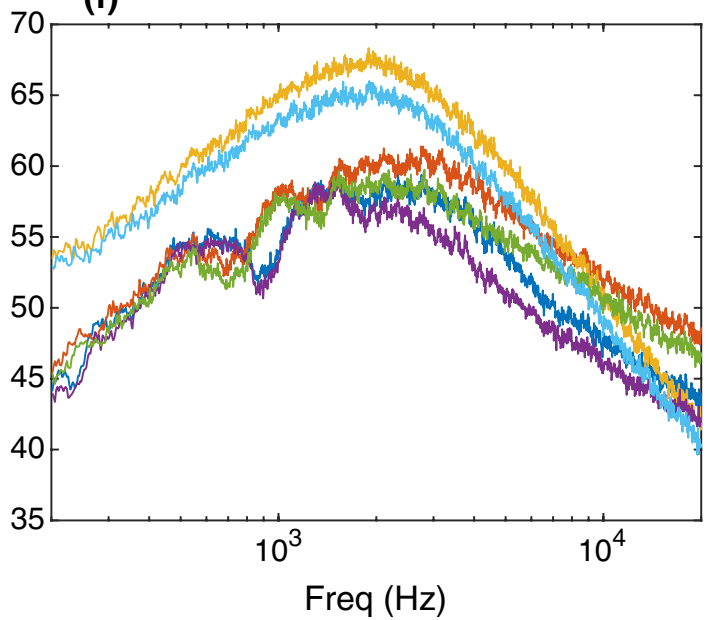

Fig. 8 Installed noise spectra of the round and lobed jets on the shielded side for various plate positions at the Mach number of 0.7 : a $L=6 D, H=3 D ; \mathbf{b} L=6 D, H=2 D ; \mathbf{c} L=6 D, H=1.5 D ; \mathbf{d} L=4 D, H=2 D ; \mathbf{e} L=4 D, H=1.5 D ; \mathbf{f} L=4 D, H=1.25 D$

be suppressed, the mode 0 still remains roughly the same. And if mode 0 is the dominant instability mode, which is known to be true for isolated jets (Suzuki and Colonius 2006; Jordan and Colonius 2013; Lyu et al. 2017), an insignificant change of the installed jet noise at low frequencies can be expected. This is in agreement with the experimental results reported in this paper.

One may wonder however, although not dominant, how are the instability waves of higher orders changed by the lobed nozzle. The earlier stability analysis also shows that 
(a)

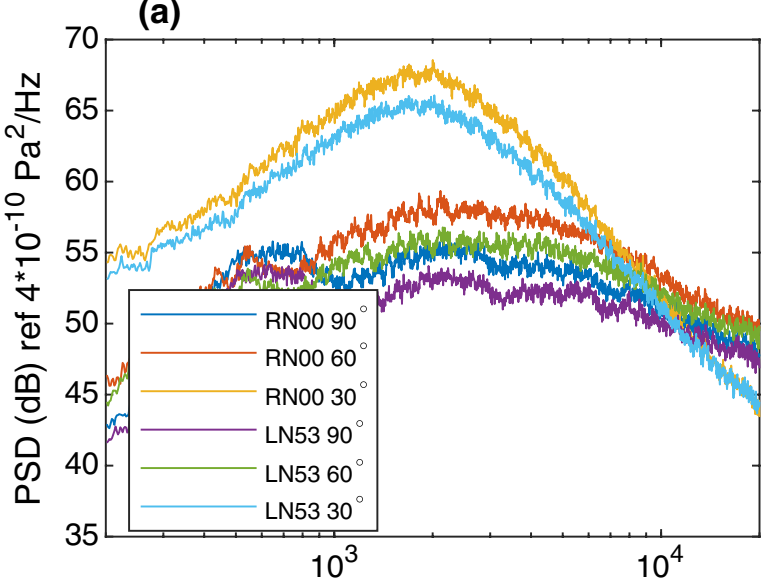

(c)

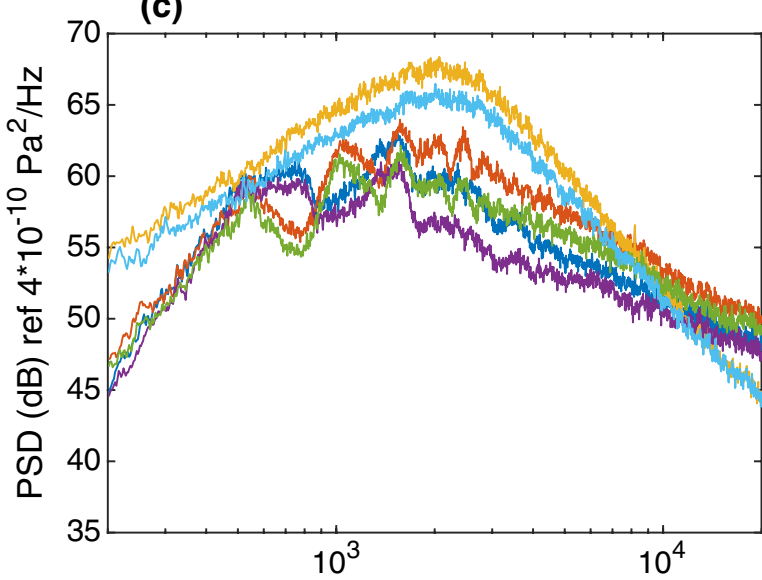

(e)



(b)

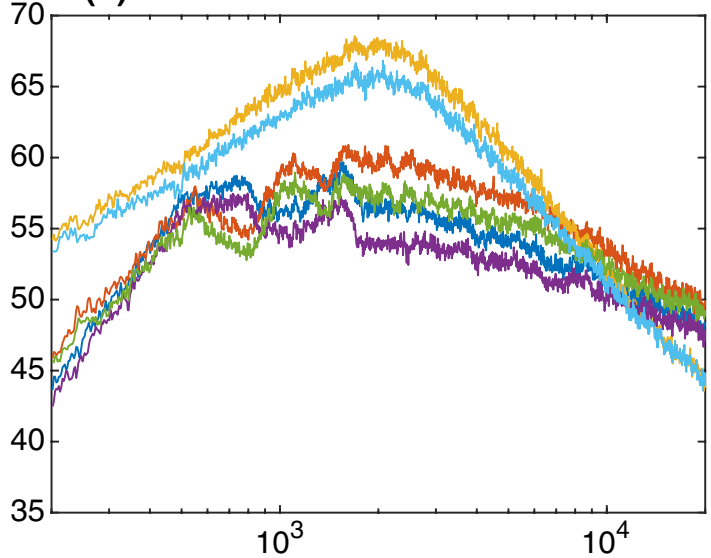

(d)

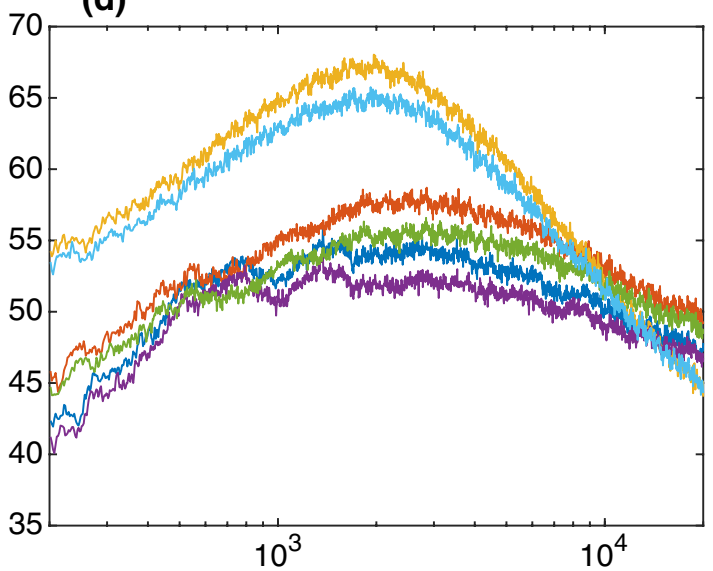

(f)

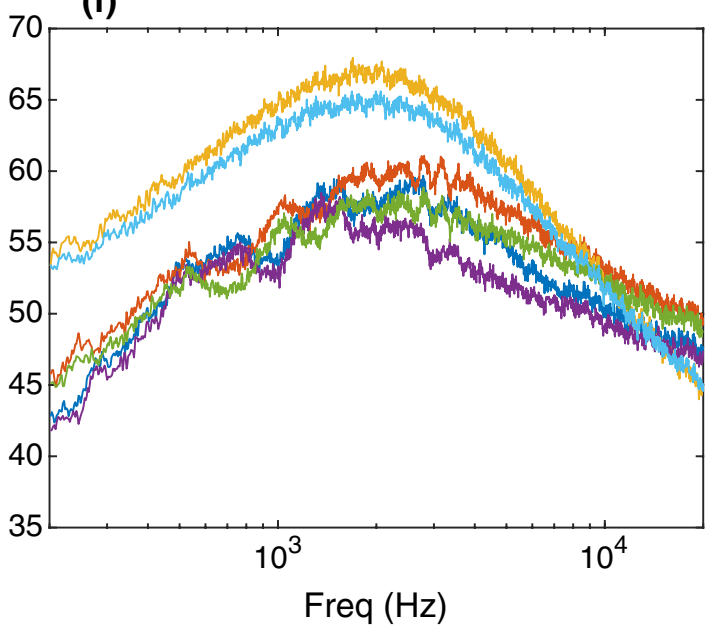

Fig. 9 Installed noise spectra of the round and lobed jets on the reflected side for various plate positions at the Mach number of 0.7 : a $L=6 D, H=3 D ; \mathbf{b} L=6 D, H=2 D ; \mathbf{c} L=6 D, H=1.5 D ; \mathbf{d} L=4 D, H=2 D ; \mathbf{e} L=4 D, H=1.5 D ; \mathbf{f} L=4 D, H=1.25 D$

a lobed vortex sheet with $N=5$ and $\epsilon=0.3$ should cause an observable increase of the convection velocity for instability waves of modes \pm 1 and \pm 2 . It should also cause a slight reduction of the temporal growth rate. Both changes are favourable to installed jet noise reduction, since the first of which results in a less efficient scattering of instability waves into sound, and the second of which results in less strong instability waves. However, care must be taken before we make such a conclusion because the earlier analysis is based on a vortex-sheet-type jet mean flow. The realistic jet mean flow might become axisymmetric quickly due to the energetic turbulent mixing when the flat plate is not too close 
to touch the jet, which is the case for all the plate configurations in this experiment. In particular, the number of lobes used in the experiment is 5, and a large number of lobes are likely to cause a quicker mixing.

\section{Conclusion and future work}

This paper studies the effects of lobed nozzles on installed jet noise. It starts with an experimental study of the isolated and installed jet noise using lobed nozzles. It is shown that the lobed nozzle has nearly identical effects on installed jet noise for all plate positions and Mach numbers: on the shielded side of the flat plate, lobed nozzles do not noticeably change the installed jet noise spectra at low frequencies. However, it does result in a noise reduction at intermediate and high frequencies. This is thought to be caused by the combination of an enhanced jet mixing and a more effective noise shielding by the flat plate. On the reflected side of the flat plate, the noise reduction is more effective in the intermediate-frequency regime, while a negligible or a slight noise increase is observed at high frequencies. This different behaviour between the shielded and reflected sides is due to the fact that on the reflected side the flat plate does not shield noise, and the increased high-frequency noise due to enhanced jet mixing propagates to the far field. The lowfrequency humps are still hardly changed by using lobed nozzles, however, compared to the results on the shielded side there is a marginal benefit of using lobed nozzles. This is believed to have been caused by the different refraction effects of the different jet plumes between the round and lobed nozzles.

To understand why lobed nozzles have little effect on the low-frequency noise humps due to the scattering of instability waves, the temporal stability characteristics of lobed jets of vortex sheet type, shown in an earlier paper, are discussed in detail. The earlier work shows that the lobed geometry changes both the convection velocity and the temporal growth rate of the instability waves. The effects are more pronounced as the number of lobes $N$ and the penetration ratio $\epsilon$ increase. However, instability waves of different orders are affected differently by the lobes. For instance, the mode 0 is particularly insensitive to the geometry changes. Higher modes are more likely to be changed significantly when both $N$ and $\epsilon$ are sufficiently large. Based on these findings, it is postulated that the little change to the installed jet noise observed experimentally at low frequencies is likely to be due to the dominance of the mode 0 instability waves. The fact that the downstream jet mean flow becomes axisymmetric quickly could also play a role. This can be verified by performing a near-field pressure measurement and/or a velocity distribution measurement using Particle Image Velocimetry, which form part of our future work.
Acknowledgements The first author (B. Lyu) wishes to gratefully acknowledge the financial support provided by the Cambridge Trust and China Scholarship Council.

Open Access This article is distributed under the terms of the Creative Commons Attribution 4.0 International License (http://creativeco mmons.org/licenses/by/4.0/), which permits unrestricted use, distribution, and reproduction in any medium, provided you give appropriate credit to the original author(s) and the source, provide a link to the Creative Commons license, and indicate if changes were made.

\section{References}

Bastos L, Deschamps C, da Silva A (2017) Experimental investigation of the far-field noise due to jet-surface interaction combined with a chevron nozzle. Appl Acoust 127:240-249

Beutel ME, Jünger C, Klein EM, Wild P, Lackner K, Blettner M, Binder H, Michal M, Wiltink J, Brähler E, Münzel T (2016) Noise annoyance is associated with depression and anxiety in the general population-the contribution of aircraft noise. PLoS One 11:1-10

Bridges J, Brown C (2004) Parametric testing of chevrons on single flow hot jets. In: NASA Technical Memorandum 2004-213107, NASA Glenn Research Center, Cleveland, Ohio

Bushell K (1975) Measurement and prediction of jet noise in flight. In: Proceedings of 2 nd aeroacoustics conference. American Institute of Aeronautics and Astronautics. AIAA 75-461

Casalino D, Diozzi F, Sannino R, Paonessa A (2008) Aircraft noise reduction technologies: a bibliographic review. Aerosp Sci Technol 12:1-17

Cavalieri AVG, Jordan P, Wolf W, Gervais Y (2014) Scattering of wavepackets by a flat plate in the vicinity of a turbulent jet. $\mathrm{J}$ Sound Vib 333:6516-6531

Head R, Fisher M (1976) Jet/surface interaction noise-analysis of farfield low frequency augmentations of jet noise due to the presence of a solid shield. In: Proceedings of 3rd aeroacoustics conference. American Institute of Aeronautics and Astronautics. AIAA 76-502

Huss A, Spoerri A, Egger M, Röösli M (2010) Aircraft noise, air pollution, and mortality from myocardial infarction. Epidemiology 21(6):829-836

Jordan P, Colonius T (2013) Wave packets and turbulent jet noise. Annu Rev Fluid Mech 45:173-195

Karabasov SA (2010) Understanding jet noise. Philos Trans R Soc A Math Phys Eng Sci 368:3593-3608

Lighthill MJ (1952) On sound generated aerodynamically. I. General theory. Proc R Soc A Math Phys Eng Sci 211(1107):564-587

Lighthill MJ (1954) On sound generated aerodynamically. II. Turbulence as a source of sound. Proc R Soc A Math Phys Eng Sci 222:1-32

Lighthill MJ (1963) Jet noise. AIAA J 1:1507-1517

Lopera J, Ng TT, Cain A (2006) Experimental studies on jet mixing and noise control utilizing vortex shedding pins and moderately lobed nozzles. In: Proceedings of 44th AIAA aerospace science meeting and exhibit. AIAA 2006-312

Lyu B, Dowling A (2016) Noise prediction for installed jets. In: Proceedings of 22nd AIAA/CEAS aeroacoustics conference. American Institute of Aeronautics and Astronautics. AIAA 2016-2986

Lyu B, Dowling A (2017) On the mechanism and reduction of installed jet noise. In: Proceeding of 23rd AIAA/CEAS aeroacoustics conference. American Institute of Aeronautics and Astronautics. AIAA 2017-3523 
Lyu B, Dowling A (2018a) Experimental validation of the hybrid scattering model for installed jet noise. Phys Fluids 30:085102

Lyu B, Dowling A (2018b) Temporal stability analysis of jets of lobed geometry. J Fluid Mech 860:5-39

Lyu B, Dowling A (2019) Modelling installed jet noise due to the scattering of jet instability waves by swept wings. J Fluid Mech 870:760-783

Lyu B, Dowling A, Naqavi I (2017) Prediction of installed jet noise. J Fluid Mech 811:234-268

Mahashabde A, Wolfe P, Ashok A, Dorbian C, He Q, Fan A, Lukachko S, Mozdzanowska A, Wollersheim C, Barrett SR, Locke M, Waitz IA (2011) Assessing the environmental impacts of aircraft noise and emissions. Prog Aerosp Sci 47(1):15-52

Münzel T, Knorr M, Schmidt F, von Bardeleben S, Gori T, Schulz E (2016) Airborne disease: a case of a Takotsubo cardiomyopathie as a consequence of nighttime aircraft noise exposure. Eur Heart J 37(37):2844

Piantanida S, Jaunet V, Huber J, Wolf W, Jordan P, Cavalieri A (2016) Scattering of turbulent-jet wavepackets by a swept trailing edge. J Acoust Soc Am 140(6):4350-4359

Shearin JG (1983) Investigation of jet-installation noise sources under static conditions. NASA Technical Report 2181, NASA Langley Research Center, Hampton, Virgina

Suzuki T, Colonius T (2006) Instability waves in a subsonic round jet detected using a near-field phased microphone array. J Fluid Mech 565:197-226
Tam CKW (1998) Jet noise: since 1952. Theor Comput Fluid Dyn 10:393-405

Tam CKW, Viswanathan K (2008) The sources of jet noise: experimental evidence. J Fluid Mech 615:253-292

Tam CKW, Zaman KBMQ (2000) Subsonic jet noise from nonaxisymmetric and tabbed nozzles. AIAA J 38(4):592-599

Tanna HK (1977) An experimental study of jet noise part I: turbulent mixing noise. J Sound Vib 50:405-428

Way D, Turner B (1980) Model tests demonstrating under-wing installation effects on engine exhaust noise. In: Proceedings of 6th aeroacoustics conference. American Institute of Aeronautics and Astronautics. AIAA 80-1048

Zaman KBMQ, Wang FY, Georgiadis NJ (2003) Noise, turbulence and thrust of subsonic free jets from lobed nozzles. AIAA J 41(3):389-407

Zaman KBMQ, Bridges JE, Huff DL (2011) Evolution from 'tabs' to 'chevron technology' - a review. Int J Aeroacoust 10:685-709

Publisher's Note Springer Nature remains neutral with regard to jurisdictional claims in published maps and institutional affiliations. 